\title{
Factors affecting hydraulic conductivity of asphalt mixture
}

\author{
M. Aboufoul • A. Garcia
}

Received: 13 July 2016/ Accepted: 30 November 2016

(C) The Author(s) 2016. This article is published with open access at Springerlink.com

\begin{abstract}
In this paper, the topological properties of air voids in asphalt mixture: air void content, average void diameter, Euler number, genus, enclosed cavities, percolation number, aspect ratio, circularity and tortuosity were analysed using X-ray tomography scans and related to the hydraulic conductivity of a wide range of asphalt mixtures representative of those commonly used in practice. Moreover, a model for the hydraulic conductivity of asphalt mixture that is valid for the whole range of air void content was proposed. The model is based on statistical and physical considerations that lead to a system of functional equations. Finally, the model was related to experimental and literature data. It was observed that the range of asphalt mixtures studied hydraulic conductivity is related mostly to the air void content, while the topological parameters (e.g. tortuosity or aspect ratio) are not the primary factors affecting hydraulic conductivity. For this reason, the hydraulic conductivity of asphalt mixture commonly used in practice can be predicted using a simple hyperbolic equation with fixed, known, parameters.
\end{abstract}

M. Aboufoul · A. Garcia ( $\square)$

Department of Civil Engineering, Nottingham

Transportation Engineering Centre [NTEC], University of

Nottingham, Nottingham NG7 2RD, UK

e-mail: alvaro.garcia@nottingham.ac.uk
Keywords Hydraulic conductivity - Air void content $\cdot$ Asphalt mixture $\cdot \mathrm{X}$-ray CT $\cdot$ Weibull model · Functional equations

\section{Introduction}

Hydraulic conductivity of asphalt mixture has a substantial impact on the ability of roads to infiltrate the surface water to the sublayers. Asphalt with high permeability will reduce the surface runoff quantity [1] and increase traffic security. However, an excess of water retained in asphalt may result in pavement distress through moisture damage, i.e. losing the bond between aggregates and the binder [2]. Moisture damage is associated with stripping, excessive permanent deformation and cracking [3, 4]. Therefore, predicting the hydraulic conductivity and understanding the factors influencing it will help to establish a balance between deterioration and drainage performance.

Hydraulic conductivity of saturated asphalt mixture is defined as the rate of discharge flow of water through the cross unit area under laminar flow conditions [5]. Its value provides an indication of the drainage capacity of asphalt mixture pavements. In addition, hydraulic conductivity is considered to be anisotropic; consequently, the hydraulic conductivity in the vertical and horizontal directions usually presents significative differences [6], although the reason for this could be that the methods to measure vertical and horizontal hydraulic conductivities differ. 
Besides, different authors have stated that hydraulic conductivity increases with the air void content and pore connectivity [7], while it is reduced with the aspect ratio of air void and tortuosity [8]. The relationship between all these topological parameters and hydraulic conductivity is not clear yet.

On the other hand, previous studies have outlined the influence of macropores on the hydraulic conductivity of soils. Representative examples are Katuwal et al. [9, 10], who found that pore connectivity and pore size distribution are vital for the flow of air and water through soils. Luo et al. [11-13] found that macroporosity (air void content) and number of connected paths are the best predictors for the hydraulic conductivity of soils. Moreover, Naveed et al. [14] found a linear relationship between air permeability and macroporosity of soils.

Today, there are different equations to predict the hydraulic conductivity of asphalt mixture, such as: (1) Kozeny-Carman equation [15], which does not apply to asphalt mixtures with hydraulic conductivities less than $1 \times 10^{-1} \mathrm{~cm} / \mathrm{s}$ [7]. (2) Exponential [16] power [17] and hyperbolic [18] models, which are arbitrarily selected and do not have a physical or fundamental statistical basis. (3) Norambuena-Garcia model [19] that was deduced from physical principles and is not representative for mixtures with very low hydraulic conductivities [16].

Moreover, Fig. 1 presents a review of the primary experimental results [19-32] on the saturated hydraulic conductivity of asphalt mixtures, measured using multiple tests and under different conditions. The conclusion obtained from Fig. 1 is that the hydraulic conductivity has a clear relationship with the air void content, following a positive trend. On the other hand, results present a high dispersion, possibly for the variety of techniques used to measure hydraulic conductivity and air void content.

The main objective of this research is to understand the effect of air void topology on the hydraulic conductivity of asphalt mixture. With this purpose, permeability tests have been conducted on asphalt test samples with a wide range of air void content, ranging from 8.7 to $26 \%$, built by changing aggregate gradation and using different compaction levels. We have aimed that these materials are representative of asphalt mixture in practice. Furthermore, CT-scans have been used to assess the structure of air voids, and their topological properties have been related to the hydraulic conductivity of asphalt mixture. Finally, a Weibull model for hydraulic conductivity has been proposed and used to explain the relationship between hydraulic conductivity and air voids in commonly used asphalt road materials.

\section{Experimental method}

\subsection{Description of materials}

Three sets of asphalt mixtures were produced; see Table 1 for the aggregate gradation and binder content
Fig. 1 Hydraulic conductivity versus air void content of the asphalt mixtures in the literature [19-32]

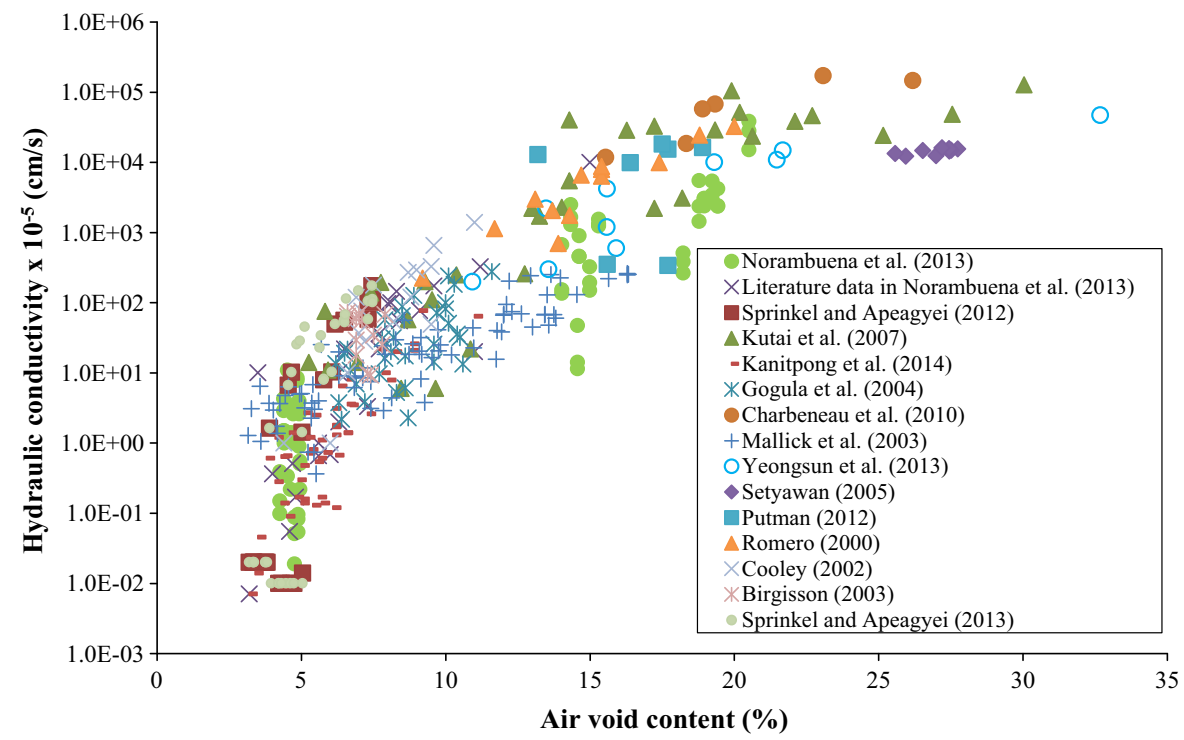


Table 1 Asphalt mixture composition

\begin{tabular}{|c|c|c|c|c|c|c|c|}
\hline \multirow[t]{3}{*}{ Target air void content sieve size $(\mathrm{mm})$} & \multicolumn{7}{|c|}{ Cumulative aggregate weight $\%$ passing } \\
\hline & \multicolumn{5}{|c|}{ Set 1 and 2} & \multicolumn{2}{|l|}{ Set 3} \\
\hline & $10 \%$ & $13 \%$ & $17 \%$ & $21 \%$ & $26 \%$ & $\begin{array}{l}(17 \%) \\
\text { Max size } 14 \mathrm{~mm}\end{array}$ & $\begin{array}{l}(17 \%) \\
\text { Max size } 28 \mathrm{~mm}\end{array}$ \\
\hline 63 & 100 & 100 & 100 & 100 & 100 & 100 & 100 \\
\hline 40 & 100 & 100 & 100 & 100 & 100 & 100 & 100 \\
\hline 31.5 & 100 & 100 & 100 & 100 & 100 & 100 & 100 \\
\hline 20 & 99 & 99 & 99 & 100 & 99 & 100 & 92 \\
\hline 16 & 91 & 91 & 91 & 96 & 91 & 100 & 84 \\
\hline 14 & 83 & 83 & 82 & 88 & 78 & 93 & 76 \\
\hline 10 & 59 & 56 & 51 & 53 & 28 & 50 & 51 \\
\hline 8 & 49 & 44 & 39 & 36 & 18 & 37 & 38 \\
\hline 6.3 & 41 & 34 & 29 & 23 & 14 & 29 & 29 \\
\hline 4 & 30 & 25 & 21 & 18 & 11 & 22 & 21 \\
\hline 2.8 & 25 & 21 & 19 & 17 & 11 & 19 & 19 \\
\hline 2 & 21 & 18 & 16 & 14 & 9 & 16 & 16 \\
\hline 1 & 15 & 13 & 11 & 10 & 7 & 12 & 11 \\
\hline 0.5 & 11 & 10 & 9 & 8 & 5 & 9 & 9 \\
\hline 0.25 & 9 & 8 & 7 & 6 & 4 & 7 & 7 \\
\hline 0.125 & 7 & 6 & 6 & 5 & 4 & 6 & 6 \\
\hline 0.063 & 6 & 5 & 5 & 4 & 3 & 5 & 5 \\
\hline Binder content $(\%)$ & 4.5 & 4.2 & 3.8 & 3.3 & 3.2 & 3.8 & 3.8 \\
\hline
\end{tabular}

used in the mixtures. Asphalt mixture in the three sets aimed to represent commonly used asphaltic materials used for roads. First, ten slabs of $300 \mathrm{~mm} \times$ $300 \mathrm{~mm} \times 50 \mathrm{~mm}$ were made with $60 / 40$ penetration grade bitumen and limestone aggregates with $20 \mathrm{~mm}$ maximum size. These materials were mixed at $160{ }^{\circ} \mathrm{C}$, and roller-compacted at $140{ }^{\circ} \mathrm{C}$ to the target air void contents: $10,13,17,21$, and $26 \%$. Second, a new set of four asphalt slabs were built using the previous aggregate gradation and bitumen content of asphalt mixture with air void content $17 \%$. These materials were also roller compacted to the target air void contents: 10, 13, 21 and 26\%. Third, two new slabs with target air void content $17 \%$ were designed with maximum aggregate size $14 \mathrm{~mm}$ and $28 \mathrm{~mm}$. Finally, 80 cores of $10 \mathrm{~cm}$ in diameter and $5 \mathrm{~cm}$ in height were extracted from the 16 slabs, five cores per slab.

\subsection{Density}

Asphalt mixture density was determined according to BS EN 12697, part 5 (2009) [33] by the mathematical method (dry mass divided by the measured volume of test samples). In addition, the bulk density of the test specimens was determined according to BS EN 12697, part 6 (2012) [34] by measuring the Bulk DensitySealed specimen (dry mass divided by the volume of test specimens calculated as dry minus submerged mass).

\subsection{Air void content $\left(A_{\mathrm{vc}}\right)$}

The air void content of asphalt mixture was calculated based on the maximum and bulk densities, as it is shown in Eq. (1).

$V_{\mathrm{m}}=\frac{\rho_{\mathrm{m}}-\rho_{\mathrm{b}}}{\rho_{\mathrm{m}}} \times 100 \%$

where $V_{\mathrm{m}}$ is the air void content in the mixture $(\%), \rho_{\mathrm{m}}$ is the maximum density of the mixture $\left(\mathrm{kg} / \mathrm{m}^{3}\right)$ and $\rho_{\mathrm{b}}$ is the bulk density of the test sample in $\left(\mathrm{kg} / \mathrm{m}^{3}\right)$.

\subsection{Hydraulic conductivity measurements}

The hydraulic conductivity test was done at room temperature $\left(20^{\circ} \mathrm{C}\right)$, following the Florida Method [35] that is a falling head method. 
Figure 2 shows the apparatus and experimental setup used to measure hydraulic conductivity of asphalt concrete cores. First, the test samples were saturated for $12 \mathrm{~h}$ by placing them under water. Then, the samples side was tightly wrapped using a latex membrane $(10 \mathrm{~cm})$. Finally, to confine the test samples and prevent the breakage of the latex membrane, the cores were confined in a hollow rubber cylinder and the gaps were sealed using silicon. Then, the rubber cylinder was placed in a hollow metal cylinder with a hole in the bottom to allow water movement, as shown in Fig. 2.

Finally, a graduated acrylic tube was placed on top of the rubber cylinder for measuring the time that $500 \mathrm{ml}$ of water needed to pass through the test samples.

The permeability coefficient $(k)$ was calculated based on Darcy's law [36] as:

$k=\frac{a L}{A t} \times \ln \left(\frac{h 1}{h 2}\right)$

where $a$ is the inner cross-sectional area of the graduated tube $\left(\mathrm{cm}^{2}\right), L$ is the test sample thickness $(\mathrm{cm}), A$ is the test sample cross-sectional area $\left(\mathrm{cm}^{2}\right), t$ is the time elapsed between the initial head and the final head (s), $h 1$ is the initial head across the test specimen $(\mathrm{cm}), h 2$ the is final head across the test specimen $(\mathrm{cm})$.

\subsection{X-ray computed tomography (CT scans)}

Asphalt cores were scanned under dry condition using a Phoenix vltomelxL scanner; the X-ray source was

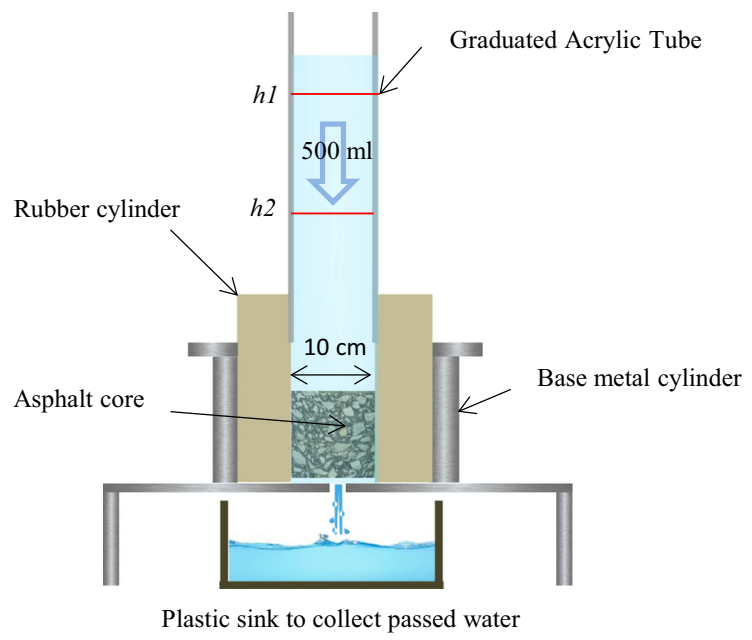

Fig. 2 Schematic representation of the hydraulic conductivity test setup operated with an acceleration voltage of $290 \mathrm{kV}$ and a current of $1300 \mu \mathrm{A}$.

The X-ray microtomography scans were carried out on the micro computed tomography Hounsfield facility at the University of Nottingham. The samples were mounted on a rotational table at a distance of $906.84 \mathrm{~mm}$ from the X-ray source. The pixel size obtained was of $96 \mu \mathrm{m}$; the scans had an isotropic resolution, meaning that the slice thickness was also $96 \mu \mathrm{m}$.

The images were originally 16-bit (.tiff format) and the voxel value represented the $\mathrm{X}$-ray attenuation. The images were processed with the software tools Avizo 8.1 and ImageJ, Version 1.49 [37], the images were converted to 8-bit grayscale resolution and cropped to a region of interest (ROI) of $6 \mathrm{~cm} \times 6 \mathrm{~cm} \times 4 \mathrm{~cm}$. 3D Gaussian and Median filters $(1 \times 1 \times 1$ Kernel size) were used to reduce the noise in the images.

Reconstructions of the microstructure were prepared by segmenting the materials in the ROI, based on grayscale thresholding. This allows separating aggregates, bitumen and air voids. With this purpose ImageJ was used.

The thresholded images of the air voids were stacked in ImageJ. Therefore, it is possible to generate surfaces that encase each group of neighboring pixels belonging to a common void space. As it is known that small isolated clusters of voids or grain voxels may correspond to small isolated pores or to noise effects [38], these were removed from the image prior further analyses. All features less than $0.5 \mathrm{~mm}$ in diameter were removed from the binary segmented data to prevent being classified as pores.

\subsection{Topology of air voids}

The macropore characteristics that were quantified based on CT Scans included macroporosity, average void diameter, connectivity, and tortuosity. These are commonly used properties to analyze the topology or soils and porous media [10]. The macroporosity was calculated as the volume of macropores per unit of volume in the ROI. Note that from now on, when we refer to air void content results will have been obtained using the mathematical method (dry mass divided by the measured volume of test samples). Moreover, when we speak about macroporosity we will refer to results obtained using CT Scan images in the ROI. 
The average void diameter was calculated using a thickness algorithm within the Particle Analyser plugin in ImageJ [26]. Furthermore, the average void diameter, Avd (mm) was calculated as:

$\operatorname{Avd}=\frac{\sum_{i=1}^{n} d_{i} V_{i}}{\sum_{i=1}^{n} V_{i}}$

where $d_{\mathrm{i}}$ and $V_{i}$ are the diameter and volume of each macropore within the ROI.

The connectivity of pore networks is commonly expressed based on the Euler number $(\chi) . \chi$ is a function of the number of isolated air voids $(N)$, the number of redundant connections in the air paths, or genus $(C)$, and the number of completely enclosed cavities $(H)[39,40]$. When the Euler number is negative, is an indication that the air voids are percolated [29]. These parameters were determined with the BoneJ particle analyser plugin (Version 1.3.11) in ImageJ [41].

$x=N-C+H$

The macropores tortuosity was calculated as the ratio of the total macropore length to the total shortest distance between the ends of all macropores in the ROI. Macropore length was obtained with the Skeletonize 2D/3D and Analyse Skeleton modules in ImageJ [41], with no pruning of the dead ends.

In addition, the percolation of air voids was defined as the relationship between the volume of the biggest air void $\left(V_{\mathrm{b}}\right)$ and the total volume of air voids in the ROI. This parameter was called Percolation Number $(\mathrm{PN})$. When this value approaches 1 , it means that all the air voids are connected [42].

Also, the air void shape properties, i.e. circularity, roundness and aspect ratio, were calculated using the Analyze Particles plugin in ImageJ, by activating the shape descriptors. When the circularity, roundness, and aspect ratio of air voids are close to 1, that represents a circular air void shape, while if the aspect ratio is higher than 1 and the value of circularity and roundness are approaching to 0 , that indicates increasingly elongated air void shapes.

\subsection{Statistics}

The correlation between the various parameters studied was analysed with linear regression and described using Pearson's correlation coefficients $(r)$.
This correlation coefficient has been considered in absolute value, with values between 0 and 1; 0 being no correlation at all, and 1 a perfect correlation. As an indication, it can be said that when the Pearson's correlation coefficient ranges from 0 to 0.4 , there is a little or very low correlation; from 0.4 to 0.7 , there is a moderate correlation and from 0.7 to 1 there is a high or very high correlation [42].

\section{Results and discussion}

\subsection{Relationship between topological properties of air voids}

The topological constants for all the test samples analysed using X-ray computed tomography have been represented in Table 2 . In addition, Table 3 shows the Pearson's correlation coefficient for all the topological properties analysed. It can be seen that the average void diameter is directly related to the volume of the biggest air void in asphalt mixture $(r=0.90)$, and that the Euler number has a very high correlation to the number of air voids $(r=0.85)$ and percolation number $(r=0.84)$. For that reason, the main topological properties studied will be the average void diameter and Euler number. Furthermore, the average void diameter and Euler number have a very high correlation to the macroporosity, $r=0.91$ and 0.69 , respectively. As an example, see Fig. 3a that shows the relationship between the Euler number and macroporosity (which is consistent with previous research, see Ref. [42]), and Fig. 3b that relates the average void diameter to the macroporosity.

Moreover, in Fig. 3a it can be observed that test samples with air void content lower than $13.7 \%$ had positive Euler number, i.e. air voids percolated in the ROI when the air void content was above $13.7 \%$. See for example the difference in the air void size between test samples \#2 $\left(A_{\mathrm{vc}}, 10.6 \%\right)$ and \#5 $\left(A_{\mathrm{vc}}, 13.7 \%\right)$ in Fig. 4. Furthermore, the average percolation number increased from $65 \%$ to $93 \%$, when the average air void content went from 9.8 to $15.1 \%$ (see Table 2). Above $15.1 \%$ air void content, the percolation number increased continuously, until it reached a value of practically $100 \%$ at $23.8 \%$ air void content. This mean that almost all the air voids are connected.

Besides, the average tortuosity of air voids was approximately 1.21 for test samples with $26 \%$ air void 


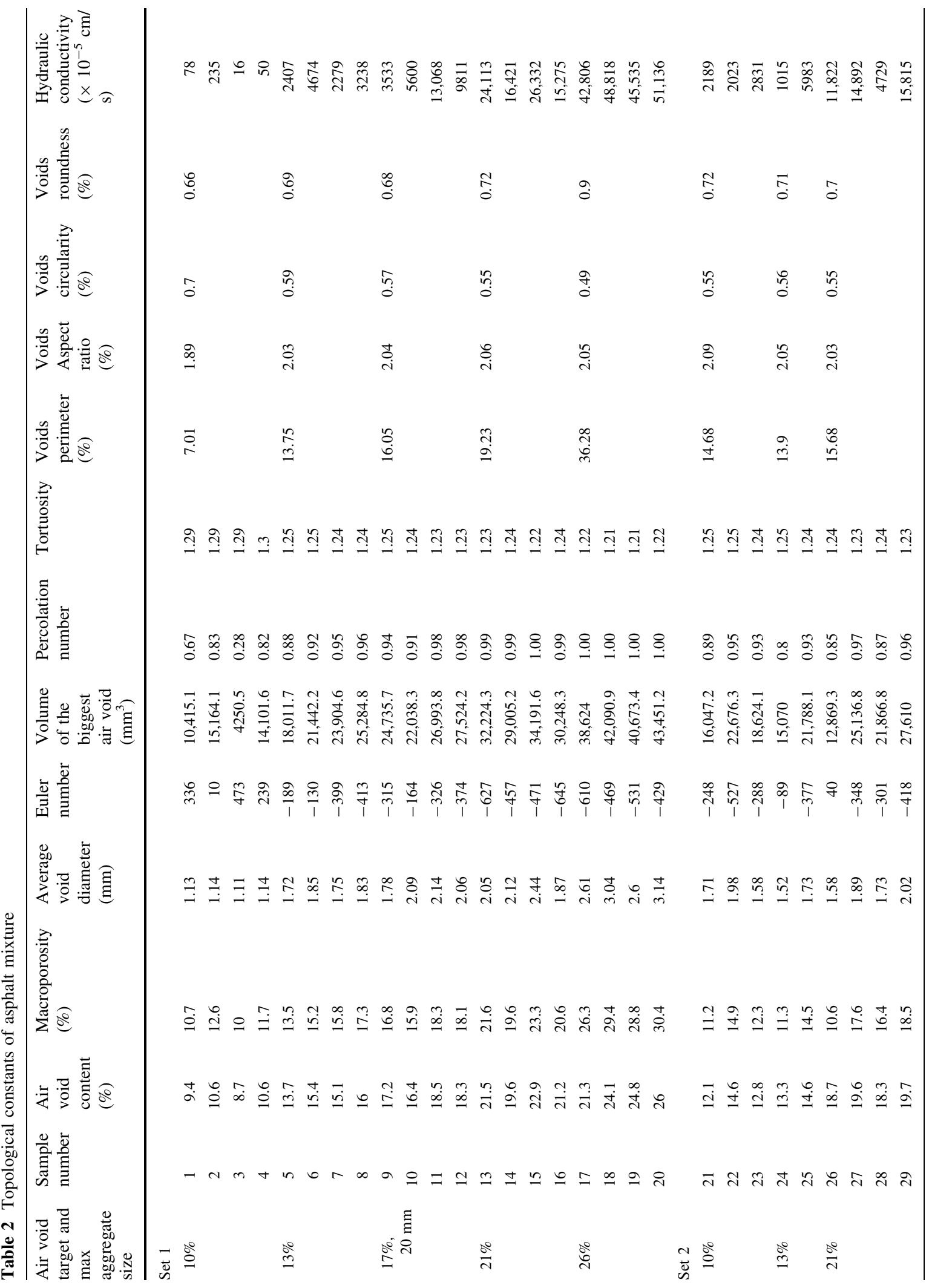




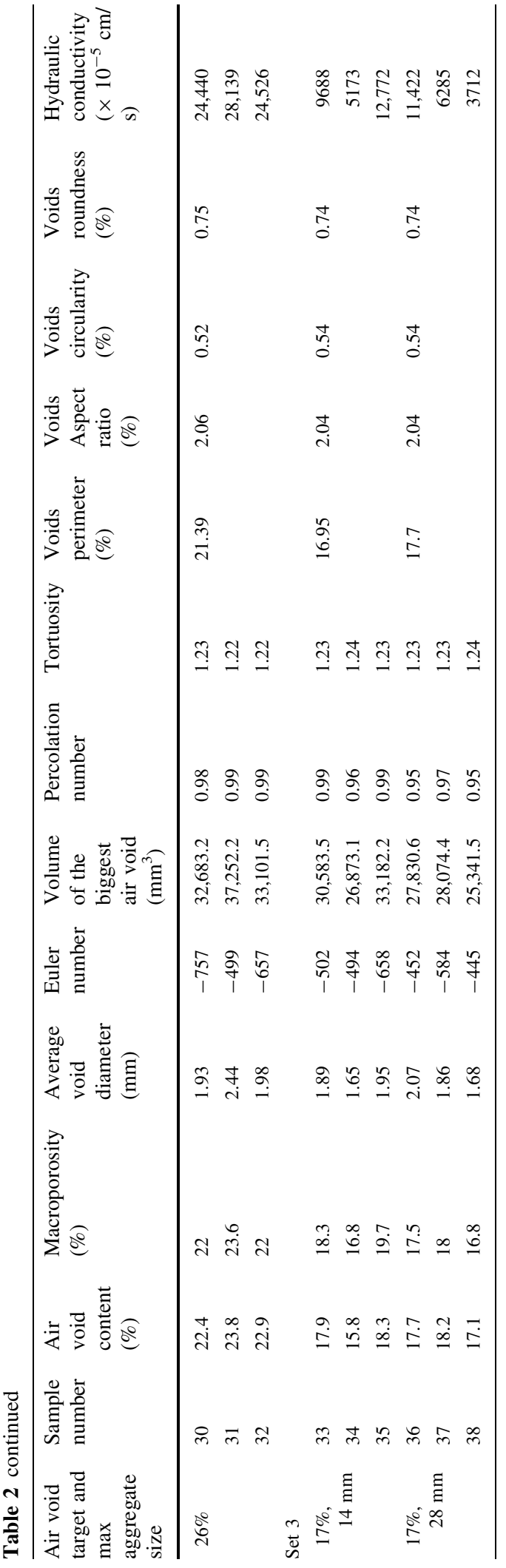

content and increased in asphalt mixture with lower air void content, until it reached the average value of 1.24 in test samples with air void content $13.7 \%$. Below this air void content, the tortuosity increased suddenly to 1.29 in test samples with $10 \%$ air void content (see Table 2). This is another indication of the percolation threshold at $13.7 \%$ air void content.

\subsection{Air void shape properties}

The average values of the air void shape properties (i.e. air void perimeter, aspect ratio, circularity and roundness) are shown in Table 2. Moreover, Fig. 5 illustrates the relationship between air void perimeter and air void content for the three sets of test samples studied. The most significant feature is that the average aspect ratio for test samples with air void content above $10.6 \%$ was approximately 2.05 and independent of the air void content, level of compaction, aggregate gradation, binder content and maximum aggregate size. As a result, the air voids were longer in the horizontal plane than in the vertical one. This effect is similar to that reported in Ref. [43] for asphalt mixture. On the other hand, the aspect ratio averaged 1.89 for test samples with air void content lower than $10.6 \%$ (see Table 2). Authors hypothesize that this happened because the air in the pores was subjected to confining pressure during compaction in mixtures with non-connected air voids.

Furthermore, Fig. 5 shows the increase in the air void perimeter with the air void content. It can be observed that above $23.8 \%$ air void content, there is a sudden increase in the air void perimeter. Furthermore, in Fig. 3a the Euler number of test samples above $23.8 \%$ air void content does not seem to increase, although more experiments are needed to validate this result. This is an indication that above $23.8 \%$ air void content, the connectivity is not improving anymore (see for example the percolation number in Table 2 for mixtures with target air void content $26 \%$ ).

In addition, Table 2 shows that the air void circularity decreases when the air void content increases. Furthermore, it shows also that there is a step reduction in the circularity when the air void content is above $10.6 \%$, as the circularity falls from 0.7 to 0.59 .

Furthermore, the air void roundness increased constantly with the air void content until it reached 
Table 3 Pearson's correlation coefficients between all the parameters studied

\begin{tabular}{|c|c|c|c|c|c|c|c|c|c|c|c|c|}
\hline & & \multicolumn{11}{|c|}{ Characteristics of air voids } \\
\hline & & $A_{\mathrm{vc}}$ & $M$ & Avd & $X$ & $C$ & $H$ & $N$ & $V_{\mathrm{b}}$ & PN & $T$ & $k$ \\
\hline Air void content & $A_{\mathrm{vc}}$ & 1 & & & & & & & & & & \\
\hline Macroporosity & $M$ & 0.91 & 1 & & & & & & & & & \\
\hline Average void diameter & Avd & 0.86 & 0.91 & 1 & & & & & & & & \\
\hline Euler number & $X$ & 0.74 & 0.69 & 0.63 & 1 & & & & & & & \\
\hline Genus & $C$ & 0.30 & 0.18 & 0.38 & 0.08 & 1 & & & & & & \\
\hline Number of enclosed cavities & $H$ & 0.24 & 0.27 & 0.15 & 0.03 & 0.56 & 1 & & & & & \\
\hline Number of air voids & $N$ & 0.33 & 0.68 & 0.77 & 0.85 & 0.43 & 0.13 & 1 & & & & \\
\hline Volume of the biggest air void & $V_{\mathrm{b}}$ & 0.91 & 0.97 & 0.90 & 0.82 & 0.15 & 0.20 & 0.79 & 1 & & & \\
\hline Percolation number & $\mathrm{PN}$ & 0.68 & 0.60 & 0.63 & 0.84 & 0.60 & 0.05 & 0.85 & 0.76 & 1 & & \\
\hline Tortuosity & $T$ & 0.87 & 0.76 & 0.83 & 0.82 & 0.37 & 0.11 & 0.94 & 0.84 & 0.76 & 1 & \\
\hline Hydraulic conductivity & $k$ & 0.86 & 0.93 & 0.88 & 0.50 & 0.24 & 0.27 & 0.54 & 0.85 & 0.44 & 0.69 & 1 \\
\hline
\end{tabular}

(a)

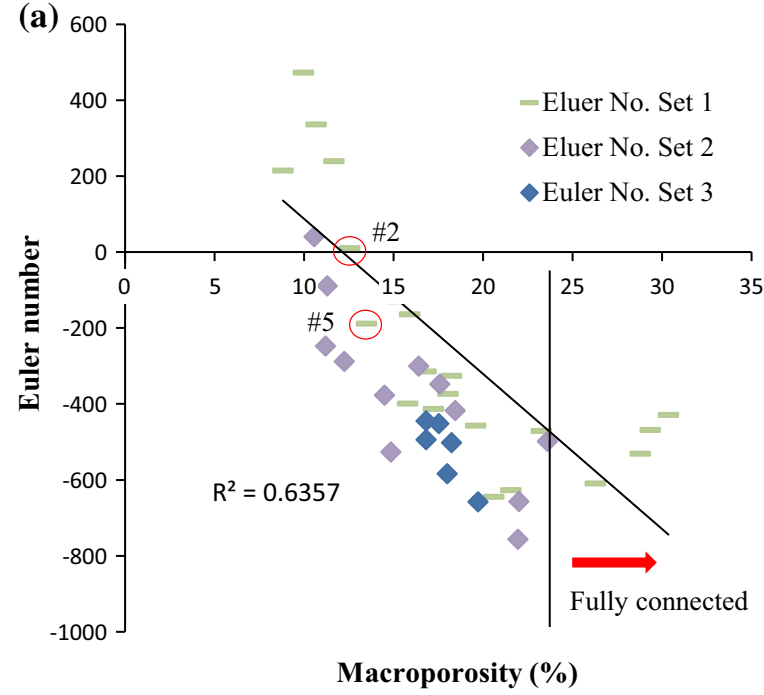

(b)

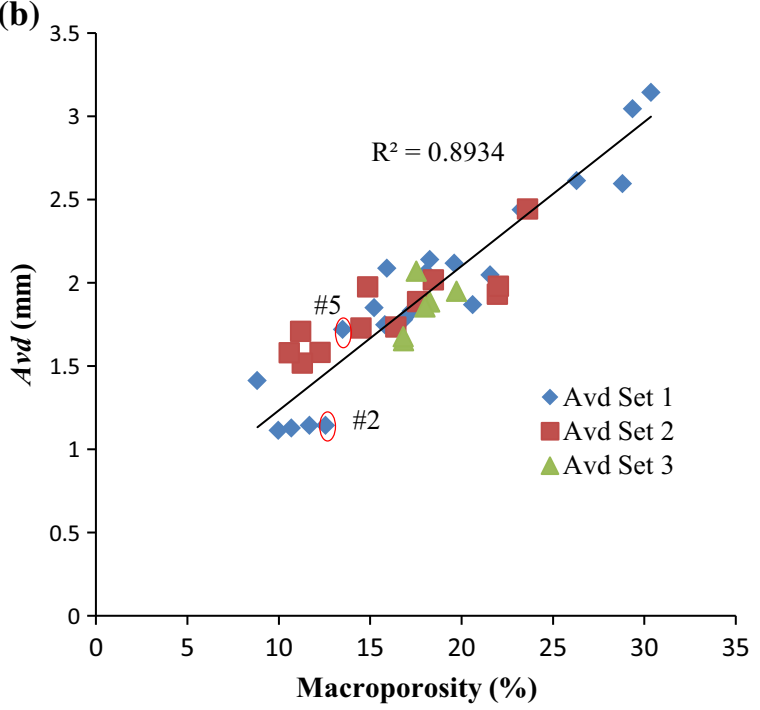

Fig. 3 Relationship between macroporosity with a Euler number for all samples studied, b average void diameter

23.8\%. At this point, the roundness and air voids perimeter increased suddenly from approximately 0.75 to 0.9 and from 25 to $40 \mathrm{~mm}$, respectively. This sudden increase of air void perimeter and roundness for test samples 18,19 and 20 could happen because asphalt mixture with $26 \%$ air void content had a much higher connectivity than the rest, which is caused by the lower amount of fine aggregates and bitumen (see Table 1). In the future more tests will be needed to validate this hypothesis.

\subsection{Hydraulic conductivity of asphalt mixture}

Figure 6 shows the hydraulic conductivity versus the air void content of all the asphalt mixtures studied. It can be seen that the hydraulic conductivity increased with the air void content, ranging from $15.9 \times 10^{-5} \mathrm{~cm} / \mathrm{s}$ at $8.7 \% A_{\mathrm{vc}}$ to $0.51 \mathrm{~cm} / \mathrm{s}$ at $26 \%$ $A_{\mathrm{vc}}$. Furthermore, Fig. 7 shows that experimental hydraulic conductivity data for test specimens with air void contents ranging between 9-11, 14-16 and 
(a)

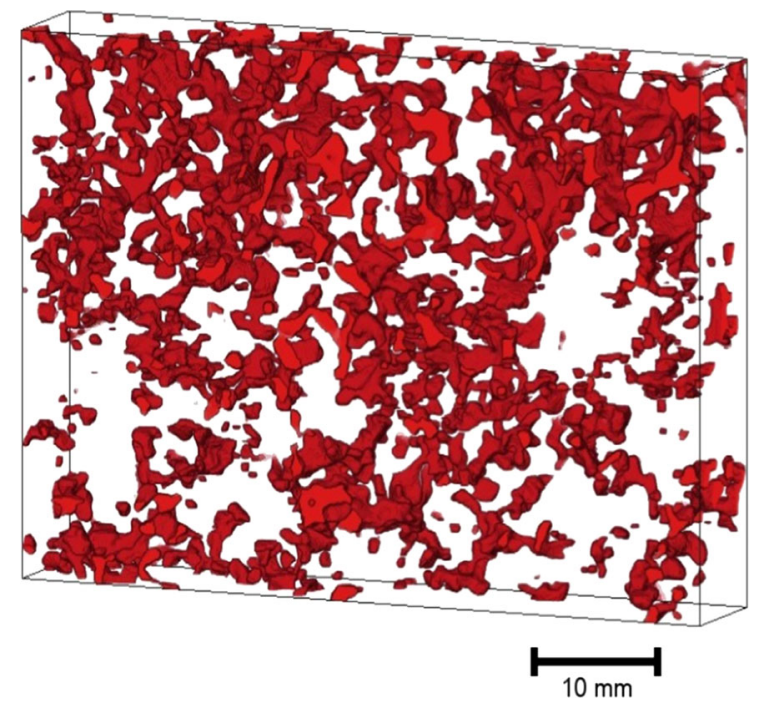

\begin{tabular}{|ll|}
\hline & \# 2 \\
$A_{V C}$ & $10.6 \%$ \\
$\mathrm{M}$ & $12.6 \%$ \\
Avd & 1.14 \\
$\mathrm{k}$ & 235 \\
\hline
\end{tabular}

(b)

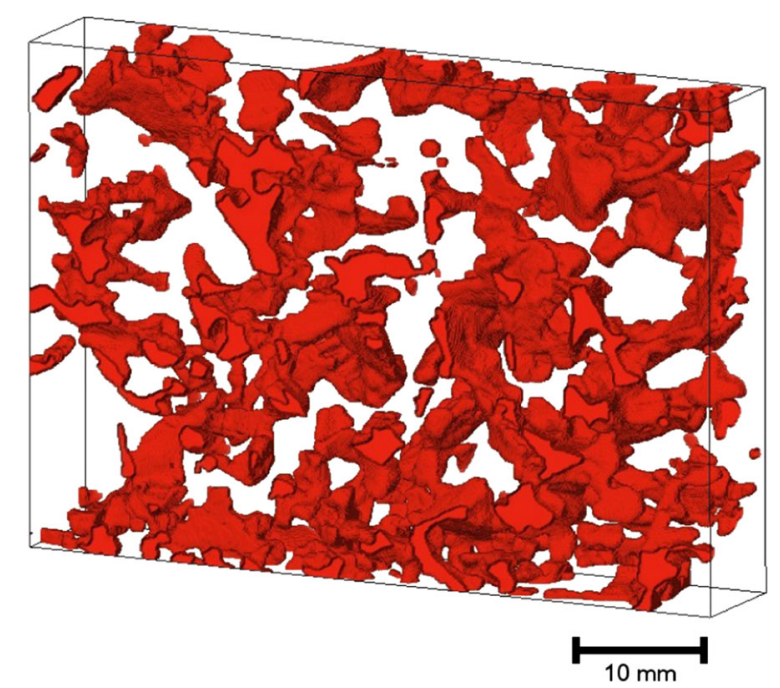

\# 5

$A_{V C} \quad 13.7 \%$

M $\quad 13.5 \%$

Avd 1.72

k 2061

Fig. 4 3D reconstruction of macropores from samples number \# 2 and \# 5

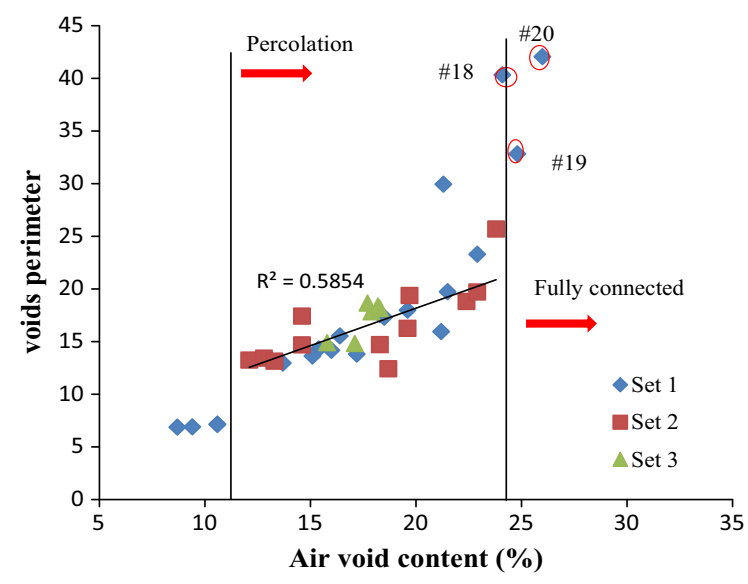

Fig. 5 Relationship between $2 \mathrm{D}$ voids perimeter properties and air void content for set 1,2 and 3

$19-21 \%$ can be successfully fitted to a Weibull distribution function. This shows that differences in hydraulic conductivity for asphalt with similar air voids are based on statistical factors.
Moreover, in Table 3 it can be seen that the main factors affecting hydraulic conductivity of asphalt mixture are (1) the average void diameter, (2) macroporosity (or air void content) and (3) volume of the biggest air void, as the Pearson's correlations are $0.88,0.93$ and 0.85 respectively. A similar result was obtained by Kumar et al. [44], although for soils, who found that saturated hydraulic conductivity had an excellent correlation with air void content. In a previous section, it has been commented that the air void content, diameter and volume of the biggest air void have a high correlation (see Table 3 ). For this reason, it can be concluded that hydraulic conductivity depends mainly on the macroporosity or air void content.

\subsection{Effect of aggregate gradation and compaction level on air voids topology and hydraulic conductivity}

Table 2 compares the average void diameter of asphalt from set 2 (i.e. built using different compaction forces) to the average void diameter from mixtures with the 
Fig. 6 Experimentally measured hydraulic conductivity versus air void content of the asphalt mixtures
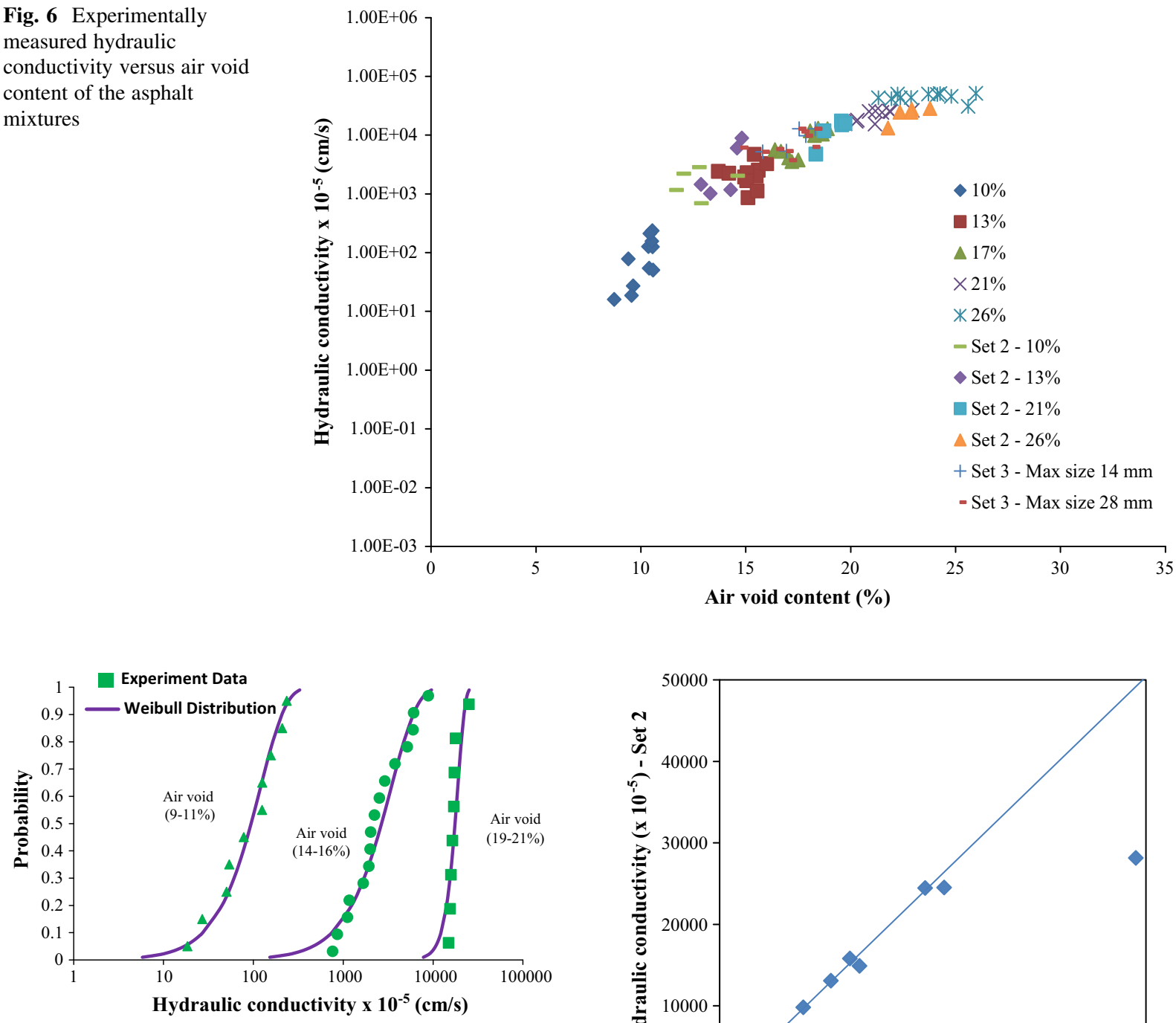

Fig. 7 Probability distribution plot of hydraulic conductivity in three air void content intervals

most similar air void content in set 1 (i.e. with different aggregate gradations). It can be seen that set 2 always had lower average void diameter than set 2 . The conclusion is that increasing or decreasing the compaction force for any mixture does not produce higher average void diameter than changing the gradation for mixtures with the same air void content. Furthermore, Table 2 shows that increasing or decreasing the level of compaction did not affect the air void aspect ratio for mixtures with similar air void content. Furthermore, Fig. 8 compares the hydraulic conductivities of test samples of set 1 and set 2 . It can be observed that when the values are compared, they align and show very similar values, which means that hydraulic

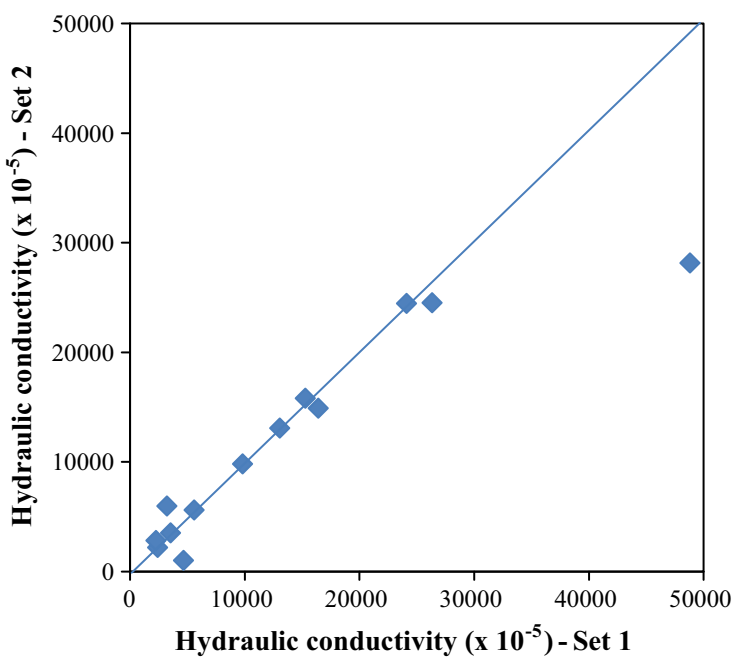

Fig. 8 Comparison of hydraulic conductivity between mixtures in set 1 and set 2

conductivity is not affected by the compaction mode of the asphalt mixture.

Finally, as it can be observed in Table 2, the air void content and diameter of the third set of test samples (i.e. constant air void content and different maximum size of aggregates) showed values independent of the maximum aggregate size. That is an indication that the maximum aggregate size had no significant effect on the hydraulic conductivity of 
asphalt mixture, although more tests have still to be conducted to confirm this point.

\section{Statistical model of hydraulic conductivity}

\subsection{Dimensional analysis}

The first step to develop a model for the hydraulic conductivity of saturated asphalt mixture is to identify the relevant variables involved. The vertical movement of water through an asphalt mixture layer of certain height will depend on (i) an arbitrarily chosen speed of water through the mixture, $k_{0}$, e.g. the maximum speed possible, when the air void content is a maximum; (ii) the speed at which water crosses the mixture, $k_{t}$; (iii) the air void content of the asphalt mixture, $A_{\mathrm{vc}}$; (iv) an arbitrary variable, $A_{v c_{0}}$, which could the maximum possible air void content in the mixture (e.g. 100\%) and (v), the probability, $p$, of having a mixture with certain hydraulic conductivity and air void content:

$v \equiv\left\{k_{t}, k_{0}, A_{\mathrm{vc}}, A_{\mathrm{vc}_{0}}, p\right\}$

The dimensional matrix is represented in Table 4. Although other variables could have been chosen, the variables in Eq. (5) have been selected for their convenience and easiness of work.

The well-known Buckingham П-Theorem allows the representation of these variables in terms of a reduced set of dimensionless variables [45]. In Table 4 it can be seen that the initial set of variables leads to a matrix of dimensions, where $L$ and $T$ refer to the fundamental magnitudes of length and time, respectively. Thus, the initial set of five variables reduces to a set of three dimensionless variables:

$f\left(\frac{k_{t}}{k_{0}}, \frac{A_{\mathrm{vc}}}{A_{\mathrm{vc}_{0}}}, p\right)=0$

In addition, we can define $A_{\mathrm{vc}} / A_{\mathrm{vc}_{0}}$ as the dimensionless air void content, $A_{\mathrm{vc}}^{*}$. $A_{\mathrm{vc}}^{*}$ cannot be negative and its value will be between 0 and $1 . A_{\mathrm{vc}}^{*}$ will be 0 when asphalt mixture is impermeable and 1 , when the

Table 4 Dimensional analysis of the initial set of variables involved in the hydraulic conductivity problem

\begin{tabular}{llllll}
\hline & $k_{t}$ & $k_{\max }$ & $A_{\mathrm{vc}}$ & $A_{\mathrm{vc}_{0}}$ & $p$ \\
\hline$L$ & 1 & 1 & 3 & 3 & 0 \\
$T$ & 1 & 1 & 0 & 0 & 0 \\
\hline
\end{tabular}

whole volume of asphalt mixture is occupied with air. Moreover, the dimensionless hydraulic conductivity, $K$, can be defined as $k_{t} / k_{o}$. Finally, Eq. (6) can be written as

$p=f\left(K, A_{\mathrm{vc}}^{*}\right)$

\subsection{Statistical derivation of the model}

First, let us imagine a very wide set of asphalt mixture test samples, with various air void contents. The mixtures are saturated and water is circulating through the air voids. $A_{\mathrm{vc}}^{*}$ and $K$ are two random variables that depend on the distribution of air voids through the mixture. In this set of asphalt mixtures, consider those with the same air void content, $a$. The probability distribution of $K$, gives the approximate percentage of the mixtures with a similar composition. In addition, their hydraulic conductivity will be $K \leq k$. Similarly, if we select a series of asphalt mixtures with a fixed $k, A_{v c}^{*}$ would also be a random variable. In this case, the air void content will be $A_{\mathrm{vc}}^{*} \leq a$. Thus, it is possible to write that $f\left(A_{\mathrm{vc}}^{*}, k\right)=f(a, K)=p$. With this in mind, it is feasible to affirm that $f\left(A_{\mathrm{vc}}^{*} \leq a \ni K=k\right)$ and $f\left(K \leq k \ni A_{\mathrm{vc}}^{*}=a\right)$.

If we build many test samples of mixtures with various air void contents, under ideal fixed conditions, e.g. always the same type of aggregates (set A), the relationship $K, A_{\mathrm{vc}}^{*}$ will look similar to the relationship in Fig. 6 . Now, let us assume that if we build a second set of mixtures (set B), by changing the gradation curve or the compaction method slightly, these mixtures will generate a slightly different $K, A_{\mathrm{vc}}^{*}$ relationship. The set A curve will not intersect with the curve generated by the Set $\mathrm{B}$ of mixture but it will be very close to it. As the $K, A_{\mathrm{vc}}^{*}$ relationships for set $\mathrm{A}$ and set $\mathrm{B}$ are not intersecting, it is possible to affirm that

$f\left(A_{\mathrm{vc}}^{*} \leq a \ni K=k\right)=f\left(K \leq k \ni A_{\mathrm{vc}}^{*}=a\right)$.

Second, we permit that $K$ and $A_{\mathrm{vc}}^{*}$ in Eq. (7) are random variables if the other is kept fixed. This is the same we have done in the previous paragraph. But now, we let the curves intersect. For this we impose the feasibility assumption:

$$
\begin{aligned}
f\left(K_{1}, A_{\mathrm{vc}_{1}}^{*}\right)= & f\left(K_{2}, A_{\mathrm{vc}_{2}}^{*}\right)=p, \text { or, } A_{\mathrm{vc}_{1}}^{*} \geq A_{\mathrm{vc}_{2}}^{*} \\
& \text { if and only if, } K_{1} \leq K_{2},
\end{aligned}
$$


If we define $A$ as the set of mixtures for which $A_{\mathrm{vc}}^{*} \leq a$, when $K=k$, and $\mathrm{B}$ is the set of mixtures for which $K \leq k$, when $A_{\mathrm{vc}}^{*}=a$, Eq. (8) will be equivalent to $\mathrm{A}=\mathrm{B}$. Now, if $a \in A$ then,

$f\left(A_{\mathrm{vc}_{a}}^{*}, K\right)=p_{a} \quad$ and $\quad A_{\mathrm{vc}_{a}}^{*} \leq a, K=k$.

In addition, when

$f\left(A_{\mathrm{vc}}^{*}, K_{a}\right)=p_{a}, \quad$ and $A_{\mathrm{vc}}^{*}=a$,

Based on the feasibility assumption (9) and on the Eqs. (10) and (11), we can conclude that for $a, K_{a} \leq k$ if $A_{\mathrm{vc}}^{*}=a$. That is $a \in b$. At the end, $\mathrm{A}=\mathrm{B}$, which validates expression (8).

Third, imagine a hypothetical mixture with all the air voids connected (e.g. mixtures with $A_{\mathrm{vc}}$ higher than 23\%). There will be a single water conduit in the mixture, which connects both extremes of the material. The mixture is composed of $n$ interconnected parallel sections. The hydraulic conductivity of the mixture, $Y$, will equal that of the section with lowest $K_{i}, 1 \leq i \leq n$. Moreover, if we let the $i$ th element be the one with lowest air void content, and thus, $K_{i}=Y$. Let $A_{\mathrm{vc}_{i}}^{*}$ be the value of the regressor variable which leads to a hydraulic conductivity $Y$, for the $i$ th element. Then, $A_{\mathrm{vc}_{i}}^{*}=X$, and

$f\left(X, A_{\mathrm{vc}_{i}}^{*}\right)=f\left(K_{i}, Y\right)$.

Since $Y \leq K_{i}$ for all $i$, from the assumption (9) it can be inferred that $X \leq A_{\mathrm{vc}_{i}}^{*}$ for all $i$. Since $X=A_{\mathrm{vc}_{i}}^{*}$ then, $X=\min \left(A_{\mathrm{vc}_{i}}^{*}\right)$.

Additionally, since $X$ given $Y$ and $Y$ given $X$ can be expressed as the minimum of identically distributed random variables, the extreme value theory can be applied [46]. The Fisher-Tippett-Gnedenko theorem $[47,48]$ establishes that there are only three types of extreme variable distributions (i) the Weibull, (ii) the Gumbel and (iii) the Fréchet. From these distributions, only the Weibull has a finite lower limit. This applies to the hydraulic conductivity of asphalt mixture, which is lower-bounded by the molecular diffusion of water through the binder.

Finally, considering Eq. (12), it can be concluded that

$$
\begin{aligned}
1 & -\exp \left\{-\left[a\left(A_{\mathrm{vc}_{i}}^{*}\right) K+b\left(A_{\mathrm{vc}_{i}}^{*}\right)\right]^{c\left(A_{\mathrm{vc}_{i}}^{*}\right)}\right\} \\
& =1-\exp \left\{-\left[d(K) A_{\mathrm{vc}_{i}}^{*}+e(K)\right]^{f(K)}\right\},
\end{aligned}
$$

where $a\left(A_{\mathrm{vc}_{i}}^{*}\right)$ and $d(K)$ are the Weibull parameters of scale, $b\left(A_{\mathrm{vc}_{i}}^{*}\right)$ and $e(K)$ are parameters defining threshold value of hydraulic conductivity and air void content, respectively and $c\left(A_{\mathrm{vc}_{i}}^{*}\right)$ and $f(K)$ are Weibull shape parameters of the cumulative distribution function.

Equation (13) is a functional equation, in which the unknowns are the six functions $a(x), b(x), c(x), d(y)$, $e(y)$ and $f(y) . x$ representing $A_{\mathrm{vc}_{i}}^{*}$ and $y$ representing $K$. Equation (13) can also be written as

$[a(x) y+b(x)]^{c(x)}=[d(y) x+e(y)]^{f(y)}$.

This functional equation has three families of solutions, already obtained by [46]:

$$
\begin{aligned}
& f(x, y)=1-\exp \left[-E(R x+S)^{C \log (a y+b)}\right]^{E}, \\
& f(x, y)=1-\exp [-C(x-A)(y-B)+D]^{E}, \\
& f(x, y)=1-\exp \left[-E(x-A)^{C}(y-B)^{D}\right] .
\end{aligned}
$$

where $A, B, C, D, E, R, S, a$ and $b$ are constants.

Any of the families (15), (16) and (17) could be used to fit hydraulic conductivity versus air voids data but the zero percentile curves of model (17) degenerate in the asymptotes. This implies that in model (17) specimens with lowest hydraulic conductivity are not affected by the air void content. For this reason, model (17) will be discarded.

In models (15) and (17), the hydraulic conductivity increases with the air void content. On the other hand, model (15) has the shape parameter variable with hydraulic conductivity, which as it can be seen in Fig. 7a is not correct, because all the points seem to align with a single model independently of the type of mixture used. For this reason, we will select the model (16) to fit hydraulic conductivity data.

Finally, forcing the percentile at some point and rearranging the constants again, $f(x, y)$ has the form

$y=A+\frac{B}{x-C}$

where $A, B$ and $C$ are constants, different than in (15), (16) and (17).

Finally, the asymptotic value of the hyperbole when the hydraulic conductivity has a vesry low value is

$\lim _{y \rightarrow-\infty} x=C$, where $B<0$, because $x \geq 0$. 
Fig. 9 Fitting of Eq. (18) to the experimental data represented in Fig. 6 and literature data

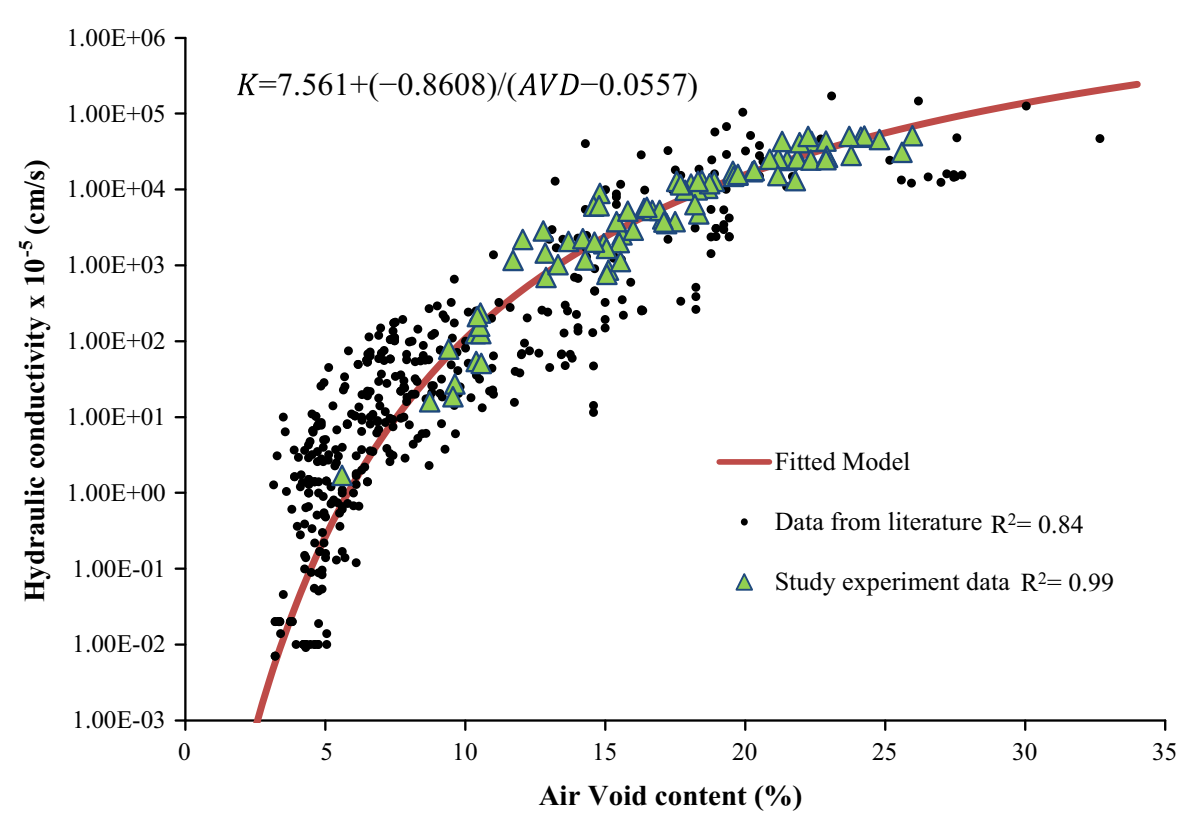

The asymptote of the hyperbole when the air void content tends to a very high value is

$\lim _{x \rightarrow-\infty} y=A$, where $A>0$.

Finally, the asymptote of the hyperbola when the hydraulic conductivity tends to 0 is

$\lim _{y \rightarrow 0} x=C-\frac{B}{A}$, where $C<0$.

4.3 Validation of the model

To validate the results, the data have been fitted to Eq. (18) using the least square method. The parameters obtained are $A=7.56, B=0.86$ and $C=0.056$. Figure 9 shows the fitting of Eq. (18) to the experimental data in Fig. 6. Moreover, the R square values are 0.99. The high $R$ square value for the experimental data confirms that the Weibull-based statistical model is appropriate to fit the experimental results. In addition, the hydraulic conductivity of asphalt mixture in the configurations studied is simply related to the air void content, and the topological properties of asphalt mixture, i.e. air voids diameter, aspect ratio, tortuosity, Euler number, etc. have a strong influence on the air void content but not on the hydraulic conductivity of asphalt mixture.

Besides, Fig. 9 shows that the model proposed has a good fitting with the literature data, $R$ squared 0.84 , although the dispersion is higher. The reason for this is that the hydraulic conductivity and air void content of each dataset were measured with a range of different techniques. To have an idea of the differences between various measuring methods the authors simply suggest to see the air void content measured with the foil method in Table 2 and observe the differences with the macroporosity, obtained using CT-Scans.

Finally, based on all the information presented in this paper, the authors have concluded that (1) hydraulic conductivity of asphalt mixture is mainly related to the air void content and (2) there is a universal relationship between the air void content and hydraulic conductivity given by Eq. (18) and the parameters at the beginning of this section. For this reason, the authors suggest that future studies on the hydraulic conductivity of asphalt mixture should be focused on quantifying the differences between different testing methods and uniformizing them, to be able to predict hydraulic conductivity accurately based only on the air void content.

\section{Conclusions}

From the results, the following conclusions have been obtained: 
- Air void content has been calculated using the foil method and X-ray tomography scans. Both methods produce approximately the same results, with an error of approximately $1.3 \%$.

- Topological parameters of air voids such as the average void diameter, Euler number, enclosed cavities, percolation number, and tortuosity are all related among them and to the air void content.

- When the air void content increases, (1) the average void diameter increases; (2) Euler number decreases; (3) enclosed cavities decrease, and (4) tortuosity decreases. Furthermore, it has been found that the air voids percolate when their content is between 10 and $15.1 \%$ and that the Euler number is a good indication of the percolation threshold when it goes from positive to negative.

- Air voids perimeter, aspect ratio, circularity and roundness are all affected by the air void content, aggregate gradation and compaction mode. For example, compacting the aggregates using higher force reduces the air void content, increases the aspect ratio of the air voids and reduces their circularity. In addition, reducing the air void content below the percolation threshold reduces the aspect ratio and increases the circularity of air voids. Finally, when the air void content is above $23.8 \%$, the perimeter of air voids seems to increase substantially, which is an indication that all the air voids are connected.

- Hydraulic conductivity of asphalt mixture seems to be mostly related just to the air void content, and not to the average void diameter, Euler number, enclosed cavities, percolation number, tortuosity, air voids perimeter, aspect ratio, circularity or roundness.

- Hydraulic conductivity can be fitted versus the air void content using a Weibull regression model based on statistical and physical considerations that lead to a system of functional equations. This model is able to fit hydraulic conductivity of every asphalt mixture for every air voids ranges considered.

- Future research on the hydraulic conductivity of asphalt mixture should focus on the differences between different measuring methods, in order to be able to correct differences with the Weibull regression equation.
Acknowledgements The authors would like to acknowledge the University of Nottingham which has funded this research through the Dean of Engineering Research Scholarship for International Excellence.

Funding This study was funded internally by the University of Nottingham.

\section{Compliance with ethical standards}

Conflict of interest The authors declare that they have no conflict of interest.

Open Access This article is distributed under the terms of the Creative Commons Attribution 4.0 International License (http:// creativecommons.org/licenses/by/4.0/), which permits unrestricted use, distribution, and reproduction in any medium, provided you give appropriate credit to the original author(s) and the source, provide a link to the Creative Commons license, and indicate if changes were made.

\section{References}

1. Hassan NA, Abdullah NAM, Shukry NAM, Mahmud MZH, Yunus NZM, Putrajaya R, Hainin MR, Yaacob H (2015) Laboratory evaluation on the effect of clogging on permeability of porous asphalt mixtures. J Teknol 76(14):77-84

2. Shah BD (2004) Evaluation of moisture damage within asphalt concrete mixes. Doctoral dissertation, Texas A\&M University

3. Huang S-C, Robertson RE, Branthaver JF, Claine Petersen J (2005) Impact of lime modification of asphalt and freezethaw cycling on the asphalt-aggregate interaction and moisture resistance to moisture damage. J Mater Civ Eng 17(6):711-718

4. Apeagyei AK, Grenfell JR, Airey GD (2014) Moisture-induced strength degradation of aggregate-asphalt mastic bonds. Road Mater Pavement Des 15(Suppl. 1):239-262

5. ASTM-D5084-03 (2004) Standard test methods for measurement of hydraulic conductivity of saturated porous materials using a flexible wall permeameter

6. Harris C, Wang L, Druta C, Tan Y, Zhou G, Zhu T, Cooley L Jr (2011) Effect of permeameter size and anisotropy on measurements of field pavement permeability. Transp Res Rec 2209:41-51

7. Kutay ME (2005) Modeling moisture transport in asphalt pavements. University of Maryland, Baltimore

8. Walsh JB, Brace W (1984) The effect of pressure on porosity and the transport properties of rock. J Geophys Res Solid Earth 89(B11):9425-9431

9. Katuwal S, Norgaard T, Moldrup P, Lamandé M, Wildenschild D, de Jonge LW (2015) Linking air and water transport in intact soils to macropore characteristics inferred from X-ray computed tomography. Geoderma 237:9-20

10. Katuwal S, Moldrup P, Lamandé M, Tuller M, De Jonge LW (2015) Effects of CT number derived matrix density on preferential flow and transport in a macroporous agricultural soil. Vadose Zone J. doi:10.2136/vzj2015.01.0002 
11. Luo L, Lin H, Halleck P (2008) Quantifying soil structure and preferential flow in intact soil using X-ray computed tomography. Soil Sci Soc Am J 72(4):1058-1069

12. Luo L, Lin H, Li S (2010) Quantification of 3-D soil macropore networks in different soil types and land uses using computed tomography. J Hydrol 393(1):53-64

13. Luo L, Lin H, Schmidt J (2010) Quantitative relationships between soil macropore characteristics and preferential flow and transport. Soil Sci Soc Am J 74(6):1929-1937

14. Naveed M, Moldrup P, Arthur E, Wildenschild D, Eden M, Lamandé M, Vogel H-J, De Jonge LW (2013) Revealing soil structure and functional macroporosity along a clay gradient using X-ray computed tomography. Soil Sci Soc Am J 77(2):403-411

15. Carrier WD III (2003) Goodbye, Hazen; Hello, KozenyCarman. J Geotech Geoenviron Eng 129(11):1054-1056

16. Cooley LA, Brown ER, Maghsoodloo S (2001) Development of critical field permeability and pavement density values for coarse-graded Superpave pavements. National Center for Asphalt Technology, Auburn

17. Kanitpong K, Benson C, Bahia H (2001) Hydraulic conductivity (permeability) of laboratory-compacted asphalt mixtures. Transp Res Rec 1767:25-32

18. Nataatmadja A (2010) The use of hyperbolic function for predicting critical permeability of asphalt. In: ARRB Conference, 24th, 2010

19. Norambuena-Contreras J, Izquierdo EA, Castro-Fresno D, Partl MN, Garcia Á (2013) A new model on the hydraulic conductivity of asphalt mixtures. Int $\mathrm{J}$ Pavement Res Technol 6(5):488

20. Birgisson B, Roque R, Page GC (2003) Evaluation of water damage using hot mix asphalt fracture mechanics (with discussion). J Assoc Asph Paving Technol 72:424-462

21. Charbeneau RJ, Klenzendorf JB, Barrett ME (2010) Methodology for determining laboratory and in situ hydraulic conductivity of asphalt permeable friction course. J Hydraul Eng 137(1):15-22

22. Cooley LA, Prowell BD, Brown ER (2002) Issues pertaining to the permeability characteristics of coarse-graded Superpave mixes. Asph Paving Technol 71:1-29

23. Gogula A, Hossain M, Romanoschi SA (2004) A study of factors affecting the permeability of superpave mixtures in Kansas. Kansas Department of Transportation, Kansas

24. Jang Y, Kim D, Mun S, Jang B (2013) Proposal for the estimation of the hydraulic conductivity of porous asphalt concrete pavement using regression analysis. Int J Highw Eng 15(3):45-52

25. Kanitpong K, Bahia H, Benson C, Wang X (2003) Measuring and predicting hydraulic conductivity (permeability) of compacted asphalt mixtures in the laboratory. In: 82nd Annual Meeting of the Transportation Research Board, Washington, DC, 2003

26. Kutay ME, Aydilek AH, Masad E, Harman T (2007) Computational and experimental evaluation of hydraulic conductivity anisotropy in hot-mix asphalt. Int J Pavement Eng 8(1):29-43

27. Mallick RB, Cooley LA, Teto MR, Bradbury RL, Peabody D (2003) An evaluation of factors affecting permeability of Superpave designed pavements. National Center for Asphalt Technology, Report:03-02
28. Putman BJ (2012) Evaluation of open-graded friction courses: construction, maintenance, and performance. Rep No FHWA-SC-12-04, Clemson Univ, South Carolina Dept of Transportation

29. Romero P (2000) Laboratory evaluation of the PQI model 300. Report to FHWA

30. Setiawan A (2009) Design and properties of hot mixture porous asphalt for semi-flexible pavement applications. Media Tek Sipil 5(2):41-46

31. Sprinkel MM, Apeagyei AK (2013) Evaluation of the installation and initial condition of rosphalt overlays on bridge decks

32. Sprinkel MM, Apeagyei AK (2014) Evaluation of the installation and initial condition of thermoplastic polymermodified asphalt overlays on bridge decks. In: Transportation Research Board 93rd Annual Meeting, 2014, vol 14-3175

33. BS EN 12697 PART 5, British Standard Institute (BSI) (2009) Bituminous mixtures, test methods for hot mix asphalt: determination of the maximum density

34. BS EN 12697 PART 6, British Standard Institute (BSI) (2012) Bituminous mixtures, test methods for hot mix asphalt: determination of bulk density of bituminous specimens

35. Florida D (2004) Florida method of test for measurement of water permeability of compacted asphalt paving mixtures. FM5-565, Dept of Transportation, Tallahassee

36. FM5-565 (1997) Florida Standard Test Method FM5-565measurement of water permeability of compacted asphalt paving mixtures. Florida Department of Transportation, Tallahassee

37. Abràmoff MD, Magalhães PJ, Ram SJ (2004) Image processing with ImageJ. Biophotonics Int 11(7):36-42

38. Peth S, Horn R, Beckmann F, Donath T, Fischer J, Smucker A (2008) Three-dimensional quantification of intra-aggregate pore-space features using synchrotron-radiation-based microtomography. Soil Sci Soc Am J 72(4):897-907

39. Vogel H (1997) Morphological determination of pore connectivity as a function of pore size using serial sections. Eur J Soil Sci 48(3):365-377

40. Vogel H-J, Roth K (2001) Quantitative morphology and network representation of soil pore structure. Adv Water Resour 24(3):233-242

41. Doube M, Kłosowski MM, Arganda-Carreras I, Cordelières FP, Dougherty RP, Jackson JS, Schmid B, Hutchinson JR, Shefelbine SJ (2010) BoneJ: free and extensible bone image analysis in ImageJ. Bone 47(6):1076-1079

42. Garcia A, Hassn A, Chiarelli A, Dawson A (2015) Multivariable analysis of potential evaporation from moist asphalt mixture. Constr Build Mater 98:80-88

43. Norhidayah AH, Zul M, Mahmud H, Ramadhansyah PJ (2014) Air void characterisation in porous asphalt using X-ray computed tomography. In: Advanced Materials Research, 2014. Trans Tech Publ, pp 443-448

44. Kumar S, Anderson SH, Udawatta RP (2010) Agroforestry and grass buffer influences on macropores measured by computed tomography under grazed pasture systems. Soil Sci Soc Am J 74(1):203-212

45. Castillo E, Fernandez-Canteli A, Koller R, Ruiz-Ripoll ML, Garcia A (2009) A statistical fatigue model covering the 
tension and compression Wohler fields. Probab Eng Mech 24(2):199-209. doi:10.1016/j.probengmech.2008.06.003

46. Castillo E, Galambos J (1987) Lifetime regression-models based on a functional-equation of physical nature. J Appl Probab 24(1):160-169. doi:10.2307/3214067

47. Fisher RA, Tippett LHC (1928) Limiting forms of the frequency distribution of the largest or smallest member of a sample. Proc Camb Philos Soc 24:180-190
48. Gnedenko B (1943) The limited distribution of the maximum term of a random series. Ann Math 44:423-453. doi: $10.2307 / 1968974$ 